Working

Paper

Department

of Economics

$\mathrm{Ca}^{\prime}$ Foscari University of Venice

Andrea Attar

Dipjyoti Majumdar

Gwenaël Piaser

Nicolàs Porteiro

Common agency games with separable preferences 


\title{
Common agency games with separable preferences
}

\author{
Andrea Attar \\ IDEI, University of Toulouse \\ Dipjyoti Majumdar \\ Concordia University \\ Gwenaël Piaser \\ University of Venice \\ Nicolás Porteiro \\ Universidad Pablo de Olavide
}

\begin{abstract}
This paper examines the role of direct mechanisms in common agency games. We focus on pure strategies and deterministic contracts and show how the introduction of a separability condition on the preferences of the agent is sufficient for the Revelation Principle to hold in in this setting, when finite generic games are considered. The result goes through without imposing any restriction on the principals' payoffs. Therefore, it is still possible to restrict attention to direct mechanisms without any loss of generality even when competition over contracts is considered.
\end{abstract}

\section{Keywords}

Revelation Principle, Common Agency, Separable Preferences.

\section{JEL Codes}

D82

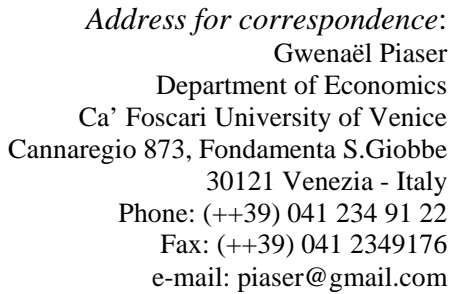

This Working Paper is published under the auspices of the Department of Economics of the Ca' Foscari University of Venice. Opinions expressed herein are those of the authors and not those of the Department. The Working Paper series is designed to divulge preliminary or incomplete work, circulated to favour discussion and comments. Citation of this paper should consider its provisional character.

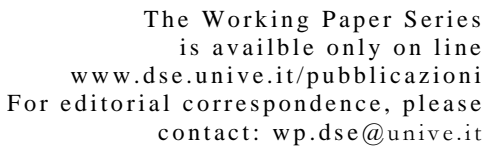

The Working Paper Series

is availble only on line www.dse.unive.it/pubblicazioni

For editorial correspondence, please

contact:wp.dse@unive.it

Department of Economics

Ca' Foscari University of Venice

Cannaregio 873, Fondamenta San Giobbe

30121 Venice Italy

Fax: ++390412349210 


\section{Introduction}

This paper studies the applicability of the Revelation Principle in common agency games. Common agency games refer to a scenario where multiple principals compete over the contract offers they make to a single agent. It involves the following timing: principals act as mechanism designers and simultaneously offer contracts to the single agent. In a next step, the agent chooses her preferred alternative, contracts are executed and the equilibrium allocation is implemented. The study of such contractual situations is receiving great attention in the recent literature. Examples include: Biais, Martimort, and Rochet (2000) who analyze non-linear pricing interactions in financial markets, Parlour and Rajan (2001) who study the competition among lenders offering non-exclusive credit contracts. Applications to lobbying are presented in Dixit, Grossman, and Helpman (1997), while Bernheim and Whinston (1998) develop an analysis of exclusive dealing agreements. ${ }^{1}$

One important issue in this class of problems is the study of communication mechanisms between the principals and the agent. In the classical one-principal one-agent (or multi-agent) problem, the study of such communication mechanisms is simplified by the celebrated Revelation Principle (Gibbard (1973); Myerson (1979)). By the Revelation Principle, every state-contingent contract induced by the Bayesian Nash Equilibrium of a communication game can be generated by a Bayesian Incentive Compatible direct mechanism. In such a scenario, the relevant private information for the principal is only the agent's "type". When more than one principal contracts with one agent, generally, such simple direct mechanisms do not reproduce all the equilibria of the underlying communication game.

The main rationale behind the failure of the Revelation Principle can be summarized as follows: at the stage of contracting with any principal the agent's private information involves both her type and the mechanisms simultaneously offered by all the other principals. Direct mechanisms are too simple as principals cannot profit from the extra information held by the agent. ${ }^{2}$

The most recent literature offers two alternative ways to face this difficulty. On the one hand, Epstein and Peters (1999) show that in multi-principal-multi-agent problems it is possible to construct a universal type space that embeds all the agents' private information: with respect to this space the Revelation Principle is still valid. In the same line, the recent work by Pavan and Calzolari (2003) introduces a Markovian Revelation Principle for common agency games. It is possible to restrict without loss of generality to mechanisms implying agent's truthful revelation of her extended type, including agent's private information, as well as all the payoff-relevant decisions contracted with the multiple principals. The main difficulty with these general solutions is the analytical complexity of the relevant mechanisms and types that limits their applicability to common contracting problems.

On the other hand, Peters (2001) and Martimort and Stole (2002), instead of looking at the relationship between indirect and direct mechanisms when multiple principals compete, emphasize how the payoffs

\footnotetext{
${ }^{1}$ In the terminology introduced by Segal and Whinston (2003), these contributions can be seen as different bidding games.

${ }^{2}$ For examples of multiprincipal games in which the Revelation Principle fail, see Peck (1997); Epstein and Peters (1999); Peters (2001) and Martimort and Stole (2002).
} 
supported by general indirect mechanisms can be as well supported as equilibria in the class of games where principals strategies are constituted by menus over the relevant alternatives. That is, they suggest an extension of the Taxation Principle (see Rochet (1986)) to a multi-principal framework: this amounts to substituting a potentially rich set of mechanisms with a simpler one, where the agent is not required to strategically reveal her private information.

Relatively less attention has been devoted to the definition of domain conditions that guarantee the Revelation Principle to hold in common agency games. ${ }^{3}$ The present paper explores such a research direction trying to identify conditions on agent's preferences that are sufficient to prove the Revelation Principle when principals compete through mechanisms. Our condition is twofold. First, we impose the agent's preferences to be separable with respect to the decision taken by the various principals. To be more precise, what we require is that the agent's preference ordering over the contract offers by one principal should not depend on those made by the others. However, as we show later with examples, the separability condition is not sufficient to guarantee the revelation principle to hold. The reason is that the agent, given a contract offer by one principal, may be indifferent between contract offers by a second principal, and this may lead to more than one equilibria, amongst which the agent is indifferent, but the principal(s) are not. This may lead to a situation where one of the equilibrium outcomes may not be supported in a direct mechanism. So we impose the following requirement: We require that the agent is never indifferent between two contract offers of each principal, for a given set of contracts offered by all other principals.

If the two requirements are satisfied, then every message sent by the agent to principal $i$ will not depend on the contract offer she is receiving from principal $j$. This enables us to reestablish the Revelation Principle. When the relevant environment is one of incomplete information (pure adverse selection), the above conditions are sufficient per se. The situation differs when the agent takes some actions, either contractible or not (i.e., if we add to the problem moral hazard considerations). As will be made clear later, in these situations we need to strengthen the conditions in appropriate ways if we are to restrict attention to direct mechanisms.

It is worth noting that no restriction on principals' preferences is introduced throughout the analysis. That is, we are able to restore the Revelation Principle even if direct externalities among principals are allowed.

The paper is organized as follows: Section 2 presents the general model; Section 3 deals with the pure incomplete information case. Sections 4 and 5 examine the scenario where the single agent is also allowed to take actions (non-contractible and contractible, respectively). Section 6 concludes and briefly relates our results to the work of Peters (2003) that investigates a similar issue.

\footnotetext{
${ }^{3}$ This line of research has originally been suggested by Martimort (1992).
} 


\section{The model}

We consider a scenario where there are $N$ principals (indexed by $i \in N=\{1,2, \ldots, N\}$ ) contracting with one agent (denoted by index, 0 ). Agent's type $\theta$ is drawn from the set $\Theta$ according to the probability distribution $F$ that is common knowledge.

Each principal $i$ is allowed to choose an action in the set of feasible allocations $Y_{i}$. An allocation can be, for example, monetary transfers, tax rates, prices, or quantities, depending on the particular interpretation of the model. The single agent can take the unobservable effort decision $e \in E$ and the action $h \in H$ that all principals can contract upon. We take the sets $\Theta, H$ and $E$ as well as the decision sets $Y_{i}$ for $i \in N$ to be finite. Each principal can hence write contracts that are contingent on the action $h \in H$ chosen by the agent. In addition, non-contractibility of $e$ introduces a standard moral hazard problem.

Players have expected utility preferences. The agent's payoff is given by the von Neumann-Morgenstern utility function $U(y, e, h, \theta)$, and for each principal $i$ the payoff is given by $V_{i}(y, e, h, \theta)$.

We consider a simple structure for communication games under incomplete information. Each principal independently communicates with the single agent selecting a communication process or mechanism. A mechanism chosen by principal $i$ is constituted by a message space $M_{i}$ and a choice rule that associates an allocation to every pair of messages and of contractible actions chosen by the agent.

Allocations are determined in the following way. First, every principal selects and offers a mechanism; mechanisms are public, in the sense that they are observed by the agent. In a next step, the agent simultaneously sends a message to each principal and takes her action decisions. More formally, principal $i$ chooses a relevant message space $M_{i} \in \mathcal{M}_{i}$ where $\mathcal{M}_{i}$ is the set, that we assume to be finite, of all feasible message spaces he could choose to receive communications from the single agent.

For the sake of expositional clarity, we follow the literature in common agency games and assume that principal $i$ 's set of feasible message spaces $\mathcal{M}_{i}$ is a singleton for $i=1,2, \ldots, N$. In other words, we take the array of message spaces $\left(M_{1}, M_{2}, \ldots, M_{N}\right)$ as given. As a consequence, a mechanism $\sigma_{i}$ chosen and offered by principal $i$ will be only identified by an allocation rule, that is, a map from $M_{i} \times H$ to $Y_{i}$. In addition, we introduce a richness assumption regarding $M$ : we should have $\Theta \subset M_{i}$ for any $i{ }^{4}$

Given $M_{i}$, a pure strategy for principal $i$ is hence the map:

$$
\sigma_{i}: M_{i} \times H \rightarrow Y_{i}
$$

We denote by $\Sigma_{i}$ the (finite) set of all strategies for principal $i$, and by $\Sigma_{N}$ the collection of all the $\Sigma_{i}$ sets. One should note that we are restricting our attention to deterministic mechanisms. Once again, this

\footnotetext{
${ }^{4}$ Both these assumptions are used in the benchmark theoretical works in common agency (see Peters (2001) and Martimort and Stole (2002)).
} 
restriction is standard in the applied literature on common agency (see Martimort and Stole (2003) and Martimort and Stole (2001)).

In common agency games, the agent's behavior depends on both her type $\theta$ and on the mechanisms $\left(\sigma_{i}\right)_{i=1}^{N}$ offered by all principals. The dependence of the message $m_{i}$ sent to principal $i$ on other principals mechanisms differentiates this setting from the standard single principal one where only valuations matter. That is, at the stage of contracting with the $i-t h$ principal, the agent has private information about her own preferences and about the contract offers she is simultaneously receiving from all the others $-i$ principals. The latter is usually referred to as market information:

When firms compete by choosing mechanisms, followed by consumers choosing firms, the type refers to the initial information about each others' characteristics, which we call the initial type, and the new information consumers have about the mechanisms chosen by firms. ${ }^{5}$

Thus, a pure strategy for the single agent is the map:

$$
\sigma_{0}: \Theta \times \Sigma_{N} \longrightarrow M \times E \times H
$$

where $M=\times_{i=1}^{N}\left(M_{i}\right)$. That is, after observing the whole array of offered mechanisms, the single agent is simultaneously choosing an effort level $e \in E$, an observable action $h \in H$ and an array of messages $m \in M$, one for each principal. We also denote by $\Sigma_{0}$ the (finite) strategy set for the agent.

Agent's participation decisions can be directly introduced in the analysis. One possible way would be to consider her choice of accepting the contract offer of each $i-t h$ principal as a binary action, that can be either contractible or not depending on the particular specification of the model. ${ }^{6}$

Hence, given the array of message spaces $M=M_{1}, M_{2}, \ldots, M_{N}$ the common agency game $\Gamma_{M}$ is:

$$
\Gamma_{M}=\left\{\Theta, \Sigma_{N}, \Sigma_{0}, U(y, e, h, \theta),\left(V_{i}(y, e, h, \theta)\right)_{i \in N}, F(.)\right\}
$$

We focus on the pure strategy Perfect Bayesian Equilibria $(P B E)$ of $\Gamma_{M}$.

Definition 1 A strategy profile $\sigma^{*}=\left(\left(\sigma_{i}^{*}\right)_{i=1}^{n}, \sigma_{0}^{*}\right) \in \Sigma_{N} \times \Sigma_{0}$ is a pure strategy PBE of $\Gamma_{M}$ if:

$$
\begin{aligned}
\forall \theta \in \Theta, \forall\left(\sigma_{i}\right)_{i \in N} \in & \Sigma_{N}(M), \\
& \sigma_{0}^{*}=\left(m^{*}, e^{*}, h^{*}\right) \in \underset{(m, h, e) \in M \times H \times E}{\arg \max } U\left(\left(\sigma_{i}\left(m_{i}, h\right)\right)_{i \in N}, h, e, \theta\right)
\end{aligned}
$$

$\forall i \in N$,

\footnotetext{
${ }^{5}$ Peck (1997), p.2

${ }^{6}$ The first interpretation is chosen, for instance, in Epstein and Peters (1999). On the other hand, Parlour and Rajan (2001) provide an example of modeling participation as a non-observable action.
} 


$$
\sigma_{i}^{*} \in \underset{\sigma_{i} \in \Sigma_{i}}{\arg \max } \int_{\theta \in \Theta} V_{i}\left(\sigma_{i}, \sigma_{-i}^{*}, e\left(\sigma_{i}, \sigma_{-i}^{*}, \theta\right), h\left(\sigma_{i}, \sigma_{-i}^{*}, \theta\right), \theta\right) d F(\theta)
$$

Observe that in the game $\Gamma_{M}$ the message spaces can be quite large. One simple mechanism is one where the message space $M_{i}$ coincides with the agent's type space $\Theta$, for any $i$. In this context, such mechanisms can be called "direct mechanisms".

When restricting to direct mechanisms, we define the strategy of principal $i$ as the map $\tilde{\sigma}_{i}: \Theta \times H \rightarrow Y_{i}$; we let $\tilde{\Sigma}_{i}$ be the strategy space for principal $i$ and $\tilde{\Sigma}_{N}$ be the collection of all such strategy profiles.

The strategy of the agent is then a map $\tilde{\sigma}_{0}: \Theta \times \tilde{\Sigma}_{N} \rightarrow \Theta^{N} \times H \times E$, and $\tilde{\Sigma}_{0}$ denotes the collection of all such maps.

Given $\Theta$, the common agency game induced by direct mechanisms is therefore the tuple:

$$
\Gamma_{\Theta}=\left\{\Theta, \tilde{\Sigma_{N}}, \tilde{\Sigma}_{0}, U(y, h, e, \theta),\left(V_{i}(y, h, e, \theta)\right)_{i \in N}, F(.)\right\}
$$

Direct mechanisms have an obvious appeal - the message spaces are simple. The question then is, when can one restrict attention to direct mechanisms? In other words, is it always the case that for any equilibrium $\sigma^{*}$ of the game $\Gamma_{M}$ there is an equilibrium $\tilde{\sigma}^{*}$ of the game $\Gamma_{\Theta}$ such that the two equilibria are outcome equivalent? For common agency games this question is typically answered in the negative. In games with multiple principals, there exist equilibria which outcomes cannot be supported in equilibrium in the corresponding direct mechanism game.

The main goal of this work is to introduce restrictions on the fundamentals of $\Gamma_{M}$ that allow one to restrict principals to the use of direct mechanisms without any loss of generality. In particular, we will follow the standard analysis of common agency games and limit our investigation to pure strategy equilibria (see Martimort and Stole (2002), Peters (2001)). In addition, we only consider deterministic mechanisms. Even though this assumption is usually introduced in both theoretical and applied common agency models, the recent result of Piaser (2005) shows that this restriction is not without loss of generality. Since our main aim is to cope with the existing literature on multi-principal games, we will henceforth refer to this "restricted" version of the Revelation Principle. ${ }^{7}$

The restrictions we introduce involve the behavior of the single agent only. As a matter of fact, we develop our approach for a specific class of games: let call it $\mathcal{G}$.

Definition 2 A common agency game $\Gamma_{M}$ belongs to the class $\mathcal{G}$ if, for every $i \in N$, for every $y_{-i}$, and for every $\theta \in \Theta$ :

$$
U\left(y_{i}, y_{-i}, e, h, \theta\right) \neq U\left(y_{i}^{\prime}, y_{-i}, e^{\prime}, h^{\prime}, \theta\right), \quad \forall y_{i}, y_{i}^{\prime}, e, e^{\prime}, h, h^{\prime} \text { for }\left(y_{i}, e, h\right) \neq\left(y_{i}^{\prime}, e^{\prime}, h^{\prime}\right) .
$$

\footnotetext{
${ }^{7}$ A similar remark is suggested by Strausz (2003) in the context of single principal, multiple agents games.
} 
That is to say, everything else equal, the agent is never indifferent to a change in the contract offer made by any of the principals. Furthermore, it is required that the best reply of the agent in terms of both the effort $e$ and the action $h$ should be unique for every given array of allocations offered by principals.

Given that we are considering finite games, this condition is generically satisfied in the payoff space of the agent.

In the remaining of the paper we consider common agency games that belong to the class $\mathcal{G}$ and we show how the introduction of a separability requirement on agent's preferences enables one to reestablish the Revelation Principle.

\section{Incomplete Information}

In the next pages we focus on games in which the agent takes neither an effort choice nor a contractible action. We define this scenario by assuming $H=E=\{\emptyset\}$. With a slight abuse of notation, we denote a principal's strategy in this pure incomplete information game by $\sigma_{i}: M_{i} \rightarrow Y_{i}$. As before, the set of strategies for principal $i$ is given by $\Sigma_{i}$ and $\Sigma_{N}=\times_{i \in N} \Sigma_{i}$. The strategy for the agent is a map $\sigma_{0}$ : $\Theta \times \Sigma_{N} \rightarrow M$.

Observe that the agent's preferences are defined over the product set of allocations offered by the principals. Given the product set structure of alternatives we shall assume that the agent's preferences are separable over each component of the set of alternatives in an appropriate sense. By "separability" we mean that the agent is able to define unambiguously her preferences over the allocations offered by principal $i$ in a way that does not depend on the contract offers made by the other principals. More formally, we state the following:

Definition 3 (Weak Separability (WS)) The agent's utility function $U$ is weakly separable in the principals' decisions for all $\theta \in \Theta$, if for all $y_{i}, y_{i}^{\prime} \in Y_{i}$ and for all $y_{-i}, y_{-i}^{\prime} \in Y_{-i}$,

$$
\left[U\left(y_{i}, y_{-i}, \theta\right)>U\left(y_{i}^{\prime}, y_{-i}, \theta\right)\right] \Longrightarrow\left[U\left(y_{i}, y_{-i}^{\prime}, \theta\right)>U\left(y_{i}^{\prime}, y_{-i}^{\prime}, \theta\right)\right]
$$

Examples of weakly separable utility functions include additively separable utility functions. However, a weakly separable utility function is not necessarily additively separable (see Fishburn (1970) for a counterexample). We note here that the separable utility assumption is common in many applied analysis (see Deaton and Muellbauer (1980)).

If the agent's utility function $U($.$) is weakly separable in the allocations offered by the principal, then$ the partial utility for the agent over the contracts offers $Y_{i}$ by principal $i$ can be defined in a natural way. Given that the offers made by the other principals are fixed at the level $\hat{y}_{-i} \in Y_{-i}$, the partial utility over $Y_{i}$ is:

$$
\hat{U}_{\hat{y}_{-i}}\left(y_{i}, \theta\right)=U\left(y_{i}, \hat{y}_{-i}, \theta\right)
$$


We start by showing some properties satisfied by equilibrium strategies when the agent has separable preferences. In particular, we show that for each $i$ and for any $\hat{y}_{-i} \in Y_{-i}$, the $i$-th component of the agent's optimal strategy maximizes her partial utility at the level $\hat{y}_{-i}$. More formally, denoting $m_{i}$ as the projection of $\sigma_{0}$ on $M_{i}$, we have the following:

Claim 1 If $\sigma_{0}^{*}=\left(m_{i}^{*}, m_{-i}^{*}\right)$ is a best reply to strategies $\left(\sigma_{i}, \sigma_{-i}\right)$, then for all $\hat{y}_{-i} \in Y_{-i}$ :

$$
m_{i}^{*} \in \underset{m_{i} \in M_{i}}{\arg \max } \hat{U}_{\hat{y}_{-i}}\left(\sigma_{i}\left(m_{i}\right), \theta\right)
$$

Proof. Suppose the claim is false. This implies that there exists a $m_{i}^{\prime} \in M_{i}$ such that $\hat{U}_{\hat{y}_{-i}}\left(\sigma_{i}\left(m_{i}^{\prime}\right), \theta\right)>$ $\hat{U}_{\hat{y}_{-i}}\left(\sigma_{i}\left(m_{i}^{*}\right), \theta\right)$. Now, from Weak Separability it follows that:

$$
U\left(\sigma_{i}\left(m_{i}^{\prime}\right), \sigma_{-i}\left(m_{-i}^{*}\right), \theta\right)>U\left(\sigma_{i}\left(m_{i}^{*}\right), \sigma_{-i}\left(m_{-i}^{*}\right), \theta\right) .
$$

This contradicts the assertion that $\sigma_{0}^{*}$ is a best reply to $\left(\sigma_{i}, \sigma_{-i}\right)$.

That is, the set of optimal messages sent to each principal $i$ does not depend on the offers made all other $-i$ principals. As a consequence, we have that:

$$
\sigma_{i}^{*}\left(m_{i}^{*}\left(\sigma^{*}, \theta\right)\right)=\sigma_{i}^{*}\left(m_{i}^{*}\left(\sigma_{i}^{*}, \theta\right)\right)
$$

That is, every $P B E$ exhibits the following property: principal $i$ 's optimal strategy is independent of principal $j$ 's mechanism. It is worth noting that this property on principals' behavior is just an implication of the separability restriction on agent's preferences.

We also remark that given the mechanism offered by principal $i$, then the message he receives from the agent depends on the agent's type $\theta$ only. Let us now stress the main implication of the (WS) assumption for games belonging to the class $\mathcal{G}$. We state it as follows.

Claim 2 Consider any collection of closed sets $B_{i} \subset Y_{i}$ and denote $\times_{i \in N} B_{i}=B$, then for every $\theta \in \Theta$; then, for all games in the class $\mathcal{G}$ such that the preferences of the agent satisfy the (WS) condition, we have that:

if

$$
\tilde{y} \in \underset{y \in B}{\arg \max } U(y, \theta)
$$

and

$$
\bar{y} \in \underset{y \in B}{\arg \max } U(y, \theta)
$$

then it must be that $\tilde{y}=\bar{y}$.

Proof. The proof of the statement is straightforward. Suppose, by contradiction, that $\tilde{y} \neq \bar{y}$; then, it must be: 


$$
U\left(\tilde{y}_{i}, \tilde{y}_{-i}, \theta\right)=U\left(\bar{y}_{i}, \bar{y}_{-i}, \theta\right)
$$

As the game belongs to the class $\mathcal{G}$,

$$
\forall i \in N, \quad \tilde{y}_{i}=\bar{y}_{i} .
$$

It follows that

$$
U\left(\tilde{y}_{i}, \bar{y}_{-i}, \theta\right) \leq U\left(\bar{y}_{i}, \bar{y}_{-i}, \theta\right)
$$

and

$$
U\left(\tilde{y}_{i}, \tilde{y}_{-i}, \theta\right) \geq U\left(\bar{y}_{i}, \tilde{y}_{-i}, \theta\right)
$$

that must be satisfied as strict inequalities given that the game is in the class $\mathcal{G}$. Suppose, as an example, that $U\left(\bar{y}_{i}, \tilde{y}_{-i}, \theta\right)=U\left(\tilde{y}_{i}, \tilde{y}_{-i}, \theta\right)$; then it must be $U\left(\bar{y}_{i}, \tilde{y}_{-i}, \theta\right)=U\left(\bar{y}_{i}, \bar{y}_{-i}, \theta\right)$ : a contradiction.

The two last inequalities, however, violate the (WS) condition, since the agent turns out to have a preference reversal with respect to $y_{i}$.

Claim 2 can be also interpreted in terms of the optimal messages chosen by the agents. Suppose that for every array of mechanisms $\left(\sigma_{i}\right)_{i \in N} \in \Sigma_{N}$, both $\bar{m}$ and $\tilde{m}$ maximize the agent's payoff $U\left(\sigma_{i}\left(m_{i}\right), \sigma_{-i}\left(m_{-i}\right), \theta\right)$ with $\bar{m} \neq \tilde{m}$. Then, it must be that $\sigma_{i}\left(\tilde{m}_{i}\right)=\bar{\sigma}_{i}\left(\tilde{m}_{i}\right)$ for every $i$. That is, the allocations offered by principals when $\tilde{m}$ and $\bar{m}$ are sent must be the same.

At this point we are in a position to state the main theorem of this section.

Theorem 1 Consider a pure incomplete information common agency game $\Gamma_{M}$ in the class $\mathcal{G}$. If the preferences of the agent satisfy the Weak Separability condition, then for every outcome that can be supported as a PBE of $\Gamma_{M} \in \mathcal{G}$, there is an equilibrium of the direct mechanism game $\Gamma_{\Theta}$ that implements the same outcome.

Proof. Let us consider a $P B E$ denoted $\sigma^{*}$ of the indirect mechanism game $\Gamma_{M}$. We will now show that the distribution over outcomes generated by the strategy profile $\sigma^{*}$ can be replicated in a direct mechanism game.

Consider the game $\Gamma_{\Theta}$ where principal $i$ 's strategy $\tilde{\sigma}_{i}: \Theta \rightarrow Y_{i}$ is defined as $\tilde{\sigma}_{i}(\theta)=\sigma_{i}^{*}\left(m_{i}^{*}\left(\sigma_{i}^{*}, \theta\right)\right){ }^{8}$ The agent's strategy is a map $\tilde{\sigma}_{0}: \Theta \times \tilde{\Sigma}_{N} \rightarrow \Theta^{N}$. Notice that the agent's best reply coincides with the one defined in $\Gamma_{M}$ when principals were using indirect mechanisms. We can then rewrite principal $i$ 's payoff as

$$
\int_{\theta \in \Theta} V_{i}\left(\tilde{\sigma}_{i}(\theta), \tilde{\sigma}_{-i}(\theta), \theta\right) d F(\theta)=\int_{\theta \in \Theta} V_{i}\left(\sigma_{i}^{*}\left(m_{i}^{*}(., \theta)\right), \sigma_{-i}^{*}\left(m_{-i}^{*}\right), \theta\right) d F(\theta) .
$$

\footnotetext{
${ }^{8}$ Observe that the direct mechanism we are constructing is incentive compatible. In a pure incomplete information scenario, we say that a direct mechanism is Incentive Compatible if and only if it is an optimal choice for the agent to report her type thruthfully.
} 
Specifically,

$$
\int_{\theta \in \Theta} V_{i}\left(\tilde{\sigma}_{i}(\theta), \sigma_{-i}^{*}\left(m_{-i}^{*}\right), \theta\right) d F(\theta)=\int_{\theta \in \Theta} V_{i}\left(\sigma_{i}^{*}\left(m_{i}^{*}\right), \sigma_{-i}^{*}\left(m_{-i}^{*}\right), \theta\right) d F(\theta) .
$$

We now show that no principal has a unilateral profitable deviation. Suppose that this is not true. Then, let us suppose that there exists a principal $i$ and a strategy $\sigma_{i}^{\prime}($.$) such that:$

$$
\int_{\theta \in \Theta} V_{i}\left(\sigma_{i}^{\prime}\left(\theta_{i}^{\prime}(\theta)\right), \tilde{\sigma}_{-i}\left(\theta_{-i}^{\prime}(\theta)\right), \theta\right) d F(\theta)>\int_{\theta \in \Theta} V_{i}\left(\tilde{\sigma}_{i}(\theta), \tilde{\sigma}_{-i}(\theta), \theta\right) d F(\theta)
$$

where $\theta_{i}^{\prime}$ and $\theta_{-i}^{\prime}$ are the agent's messages to principals $i$ and $-i$ when principal $i$ is deviating. Obviously, if principal $i$ changes his offered mechanism, then the agent's reply to this deviation will be different. But Claim 2 implies that the agent's reply to the non-deviating principals will be unique and unchanged (i.e; $\theta_{-i}^{\prime}=\theta$ ). Therefore, it follows that:

$$
\int_{\theta \in \Theta} V_{i}\left(\sigma_{i}^{\prime}\left(\theta_{i}^{\prime}(\theta)\right), \sigma_{-i}^{*}\left(m_{-i}^{*}\right), \theta\right) d F(\theta)>\int_{\theta \in \Theta} V_{i}\left(\tilde{\sigma}_{i}(\theta), \sigma_{-i}^{*}\left(m_{-i}^{*}\right), \theta\right) d F(\theta)
$$

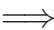

$$
\int_{\theta \in \Theta} V_{i}\left(\sigma_{i}^{\prime}\left(\theta_{i}^{\prime}(\theta)\right), \sigma_{-i}^{*}\left(m_{-i}^{*}\right), \theta\right) d F(\theta)>\int_{\theta \in \Theta} V_{i}\left(\sigma_{i}^{*}\left(m_{i}^{*}\right), \sigma_{-i}^{*}\left(m_{-i}^{*}\right), \theta\right) d F(\theta) .
$$

However this contradicts the fact that $\sigma^{*}$ is a PBE.

We can now illustrate the consequences the implications of assuming separability in agent's preferences. First consider the following game, borrowed from Martimort and Stole (2002), where the type of the agent is fixed and known.

Example 1: The agent's type space is degenerate and there are two principals: each of them can offer the three allocations $A, B$ and $C$. That is, we have: $Y_{1}=Y_{2}=\{A, B, C\}$. The payoffs of principal 1, principal 2 and the agent, $\left\{V^{1}, V^{2}, U\right\}$, are given by the following matrix:

\begin{tabular}{|c|ccc|}
\hline & $y_{2}=A$ & $y_{2}=B$ & $y_{2}=C$ \\
\hline$y_{1}=A$ & $(1,1, a)$ & $(2,0,2)$ & $(-1,5, b)$ \\
$y_{1}=B$ & $(1,2,2)$ & $(1,1,1)$ & $(0,0, c)$ \\
$y_{1}=C$ & $(5,-1, b)$ & $(0,0, c)$ & $(0,0,0)$ \\
\hline
\end{tabular}

In the original Martimort and Stole's example we have $a=1, b=10$ and $c=0$. If we consider the direct mechanism game, then there is no strategic role for the agent and the equilibrium outcomes are those that are sustained as Nash equilibria of the game played among the two principals. In particular, there is only one pure strategy Nash equilibrium: both principals play $\{C\}$ and the corresponding outcome is $(0,0,0)$.

However, if principals are allowed to communicate with the agent, then the outcome $(1,1,1)$ can also be supported at equilibrium. In particular, consider the indirect mechanism game where each principal is 
endowed with the same message space $M_{1}=M_{2}=\left\{m_{1}, m_{2}\right\}$. Now, assume that each principal chooses the following allocation rule:

$$
\begin{aligned}
& \sigma_{i}: M_{i} \rightarrow Y_{i}, \\
& \sigma_{i}\left(m_{1}\right)=B, \\
& \sigma_{i}\left(m_{2}\right)=C,
\end{aligned}
$$

for $i=1,2$.

The allocation rules $\sigma_{1}, \sigma_{2}$ are part of a Nash equilibrium. At equilibrium the agent will send the message $m_{1}$ to both principals and the outcome $(1,1,1)$ will be implemented. Principal 1 cannot profitably deviate by offering any alternative indirect mechanism because the agent is acting as a coordinating device. If he deviates by offering the mechanism:

$$
\begin{aligned}
& \sigma_{1}: M_{1} \rightarrow Y_{1}, \\
& \sigma_{1}\left(m_{1}\right)=A, \\
& \sigma_{1}\left(m_{2}\right)=A,
\end{aligned}
$$

then, the agent will send the message $m_{2}$ to principal 2 making the deviation unprofitable. The example is therefore emphasizing that there can exist outcomes implementable through indirect mechanism that are not implementable through direct mechanisms.

It is important to remark that under alternative assumptions on agent's preferences the outcome $(1,1,1)$ cannot be implemented through indirect mechanisms either. To show that, let us assume $a>2$, $b<2$ and $c<1$. In such a case, allowing for indirect mechanisms does not enlarge the set of equilibria sustainable trough direct mechanisms. For $(1,1,1)$ to be sustained as equilibrium payoffs, the utility of the agent should satisfy the following:

$$
U\left(y_{i}=B, y_{j}=B\right) \geq U\left(y_{i}=C, y_{j}=B\right)
$$

and

$$
U\left(y_{i}=B, y_{j}=A\right)<U\left(y_{i}=C, y_{j}=A\right)
$$

for every $i \neq j$.

The conditions above cannot be simultaneously fulfilled if the agent has separable preferences. Whenever the preferences of the agent are separable and he could gain by choosing $y_{i}=B$ instead of $y_{i}=C$ (given $y_{j}=B$ ), then the same has to hold when $y_{j}=A$. That is, the marginal impact on the agent's utility of a change in principal $i$ 's action has to be independent of principal $j$ 's action. The allocation $\{B, B\}$ cannot therefore be supported when preferences are separable, even if we allow principals to offer indirect mechanisms to the agent.

This first example hence provides an important hint for the analysis: existence of a preference reversal order for the agent over principals' actions is needed for indirect mechanisms to improve over direct mechanisms. This is not possible whenever preferences are separable. 
Martimort and Stole's work shows that in order to characterize the whole the set equilibria of common agency games with incomplete information it is sufficient to restrict principals to offer menus over allocations. Importantly, if preferences are separable then every allocation implementable through menus can as well implemented by direct mechanisms. We emphasize this point in a second example, still taken from Martimort and Stole (2002).

Example 2: There are two principals and one agent with type $\theta \in\{-1,1\}$, drawn with equal probability. Each principal has two actions $y_{i} \in\{0,1\}$. The utilities are $V_{1}=\theta\left(y_{1}-y_{2}\right)$ for principal 1, $V_{2}=\theta\left(y_{2}-y_{1}\right)$ for principal 2 and $U=\theta\left(y_{1}+y_{2}\right)$ for the agent. Equilibrium strategies are given by the menus $\{0,1\}$ for both principals. The outcome will always be $y_{1}=y_{2}=0($ when $\theta=-1)$ and $y_{1}=y_{2}=1$ (when $\theta=1$ ).

Observe that agent's preferences are separable in the actions of the principals and, for this reason, the same outcome can be achieved as an equilibrium of a direct mechanism game. Simply consider the following contract offers: each principal proposes the agent a direct mechanism $T_{i}$ such that:

$$
T_{i}=\left\{\begin{array}{lll}
y_{i}=0 & \text { if } & \theta=-1 \\
y_{i}=1 & \text { if } & \theta=1 .
\end{array}\right.
$$

This mechanism implements the same outcome that was supported by menus.

These two examples considered together emphasize the relationship between the set of outcomes implementable by indirect mechanisms and by direct mechanisms if the agent has separable preferences. Whenever separability and non-indifference are assumed, then every equilibrium associated to an indirect mechanism (or menu) game can be as well supported by direct mechanisms. One can legitimately ask if both conditions are necessary to get the result.

Example 3: The main aim of this example is to show that the separability requirements on the preferences of the agent are not sufficient per se to guarantee the Revelation Principle in common agency games.

We propose here a slightly modified version of our Example 1. The agent's type space is degenerate and there are two principals: each of them can offer the three allocations $A, B$ and $C$. That is, we have: $Y_{1}=Y_{2}=\{A, B, C\}$. The payoffs to principal 1, principal 2 and to the agent, $\left\{V^{1}, V^{2}, U\right\}$, are given by the following matrix:

\begin{tabular}{|c|ccc|}
\hline & $y_{2}=A$ & $y_{2}=B$ & $y_{2}=C$ \\
\hline$y_{1}=A$ & $(1,1,1)$ & $(2,1,2)$ & $(-1,5,2)$ \\
$y_{1}=B$ & $(1,2,0)$ & $(1,1,1)$ & $(0,0,1)$ \\
$y_{1}=C$ & $(5,-1,0)$ & $(0,0,1)$ & $(0,0,1)$ \\
\hline
\end{tabular}

In this game, the preferences of the agent satisfy the Weak Separability condition in a trivial sense. The Revelation Principle is, however, violated. 
First, one should notice that the outcome $(1,1,1)$ cannot be supported as an equilibrium in the direct mechanism game: both principals can profitably deviate by playing $\{A\}$.

Then, we consider the situation where the principals offer the menus $\{B, C\},\{B, C\}$ and the agent chooses the contract $B$ from both menus. The reader can check that given these menus the agent does not have a strictly profitable deviation. Moreover, consider any principal $i \in\{1,2\}$. Given that the other principal is offering the menu $\{B, C\}$, a potential profitable deviation for principal $i$ is to the degenerate menu $\{A\}$. However, if principal $i$ offers $d_{i}=A$, and principal $j$ offers the menu $\{B, C\}$, a best reply for the agent would be to choose $C$ from the non-deviating principal, and this makes principal $i$ strictly worse off. Since the game is symmetric in the principals' payoffs, the menu offers $\{B, C\}$ by both principals constitute a Nash equilibrium that supports the outcome $\{1,1,1\}$.

The key feature of the example is that the game does not belong to the class $\mathcal{G}$. It is enough to observe that if Principal 2 were playing $B$, then the agent's payoff would always be equal to one, irrespectively of Principal 1's choice. When this is the case, indirect mechanisms (and, in particular, menus) can be used to sustain outcomes that are not achievable by using direct mechanisms. Menus allow principals to strategically profit from the agent's indifference.

The last example stresses that there are games in the class $\mathcal{G}$ which do exhibit equilibria which cannot be sustained by direct mechanisms.

Example 4: Again, the agent's type space is degenerate and there are two principals: each of them can offer the three allocations $A, B$ and $C$. That is, we have: $Y_{1}=Y_{2}=\{A, B, C\}$. The payoffs to principal 1, principal 2 and to the agent, $\left\{V^{1}, V^{2}, U\right\}$, are given by the following matrix:

\begin{tabular}{|l|lll|}
\hline & $y_{2}=A$ & $y_{2}=B$ & $y_{2}=C$ \\
\hline$y_{1}=A$ & $(2,1,3)$ & $(1,2,2)$ & $(0,1,1)$ \\
$y_{1}=B$ & $(1,1,0)$ & $(1,0,5)$ & $(0,0,4)$ \\
$y_{1}=C$ & $(0,1,7)$ & $(0,0,8)$ & $(0,0,9)$ \\
\hline
\end{tabular}

First, one should notice that the outcome $(2,1,3)$ cannot be supported as an equilibrium in the direct mechanism game: If the first principal takes the decision $\{A\}$ the second principal can profitably deviate by playing $\{B\}$.

Now, if the first principal offers the menu $\{A, B\}$, the latter deviation become unprofitable. If the second principal plays $\{B\}$ rather than $\{A\}$, the agent chooses $B$ in the first principal's menu. Hence the second principal makes a payoff of 0 , then he has no reason to deviates.

The first principal has no profitable deviation, as he gets his maximal payoff. Thus, the outcome $(2,1,3)$ can be sustained as an equilibrium. This shows that the restriction to the class $\mathcal{G}$ is not sufficient to establish our version of the Revelation Principle. 


\section{Moral Hazard}

The main purpose of thois section is to extend the results obtained in the previous section to a situation where there are moral hazard considerations in addition to adverse selection factors. We assume that the single agent only takes an effort $e$ from the set $E$ (i.e. $H=\{\emptyset\}$ ), and that this effort choice is not contractible. This is the reference framework of many recent works in common agency: Bernheim and Whinston (1986a), Bisin and Guaitoli (2004), Ishiguro (2005), Martimort (2004), Parlour and Rajan (2001), Attar, Campioni, and Piaser (2005).

When there is a not contractible effort, the principals' choices are only contingent on the messages they receive. A (pure) strategy for principal $i$ is hence the map $\sigma_{i}: M_{i} \rightarrow Y_{i}$. The agent's strategy is to choose a message $m_{i}$ for each principal and an effort $e$, and we denote it $\sigma_{0}: \Theta \times \Sigma_{N} \rightarrow M \times E$. In the corresponding direct mechanism game, strategies are: $\tilde{\sigma}_{i}: \Theta \rightarrow Y_{i}$, for every principal $i$, and $\tilde{\sigma}_{0}$ : $\Theta \times \tilde{\Sigma}_{N} \rightarrow \Theta^{N} \times E$, for the agent.

In such a scenario the $(W S)$ condition on the function $U($.$) will not in general be sufficient to get the$ Revelation Principle. The reason is that the optimal action chosen by the agent depends on all the offers she is receiving from all principals; as a consequence assuming separability in $U($.$) does not guarantee$ that the agent's utility function will remain separable if we evaluate it at the optimal chosen action. Although the Revelation Principle fails for the class of separable games already defined, a strengthening of $(W S)$ retrieves it.

We refer to this condition as Strong Separability $(S S)$.

Definition 4 (Strong Separability (SS)) The agent's utility function $U$ is strongly separable in the principals' decisions $y_{i}$ 's and effort $e$ if for all $\theta \in \Theta$, for all $y_{i}, y_{i}^{\prime} \in Y_{i}$, for all $y_{-i}, y_{-i}^{\prime} \in Y_{-i}$ and for all $e^{\prime}, e^{\prime \prime} \in E$

$$
\left[U\left(y_{i}, y_{-i}, e^{\prime}, \theta\right)>U\left(y_{i}^{\prime}, y_{-i}, e^{\prime}, \theta\right)\right] \Longrightarrow\left[U\left(y_{i}, y_{-i}^{\prime}, e^{\prime \prime}, \theta\right)>U\left(y_{i}^{\prime}, y_{-i}^{\prime}, e^{\prime \prime}, \theta\right)\right]
$$

The $(S S)$ condition implies that, for each principal $i$, the agent has an unambiguous preference ordering over the contract offers made by $i$ that does not depend either on the contract offers by other principals or on the effort choice of the agent.

In a moral hazard scenario, one is allowed to define the agent's indirect utility function, that is, the utility evaluated at the optimal level of effort. For all games in the class $\mathcal{G}$ we let this utility be:

$$
U^{*}\left(y_{i}, y_{-i}, \theta\right)=\max _{e \in E} U\left(y_{i}, y_{-i}, e, \theta\right)
$$

is uniquely defined, given that $U$ is continuous and the set $E$ is closed. Importantly, if the (SS) condition holds, the utility $U^{*}$ turns out to be Weakly Separable (WS). We are therefore in the same scenario as that described in the previous section, once we consider $U^{*}$ instead of $U$. As a consequence, the same line of reasoning can be followed. In particular, the following Corollary of Theorem 1 holds: 
Corollary 1 Consider a common agency game $\Gamma_{M}$ in the class $\mathcal{G}$. If the preferences of the agent satisfy the Strong Separability condition, then for every outcome that can be supported as a PBE of $\Gamma_{M}$, there is an equilibrium of the direct mechanism game $\Gamma_{\Theta}$ that implements the same outcome.

A second issue of interest is given by those situations where the agent's actions are observable and principals can therefore offer contracts that are contingent on her choices. We deal with this issue in the next pages.

\section{Contractible Actions}

The following paragraphs analyze a scenario where for each message $m_{i}$ received from the agent, principal $i$ decides which allocation $y_{i}$ will be offered conditionally on the action $h$ chosen by the single agent. We first consider the simple framework where $h$ is fully contractible for every principal and the preference ordering of the agent is independent of her type. A particular setting that fits this framework is that in which there is complete information about the agent's preferences. This is the reference framework of many traditional works, like Bernheim and Whinston (1986b); Dixit, Grossman, and Helpman (1997); Bernheim and Whinston (1998); Laussel and Le Breton (1998) and Laussel and Le Breton (2001). In this section we show how, whenever we consider such a set-up, our separability requirement is a sufficient condition to characterize the whole set of equilibria associated to indirect mechanisms through simple direct mechanisms.

In order to adapt our notion of separability to a case where the agent's preference ordering is independent of her type, we introduce a new notion of separability. For the sake of clarity, we will refer to this condition as $\theta$-separability:

Definition 5 ( $\theta$-Separability) The utility function $U$ is $\theta$-separable in the principals' decisions $y_{i}$, for all $h \in H$, if for all $y_{i}, y_{i}^{\prime} \in Y_{i}$, for all $y_{-i}, y_{-i}^{\prime} \in Y_{-i}$ and for all $\theta, \theta^{\prime} \in \Theta$ :

$$
\left[U\left(y_{i}, y_{-i}, h, \theta\right)>U\left(y_{i}^{\prime}, y_{-i}, h, \theta\right)\right] \Longrightarrow\left[U\left(y_{i}, y_{-i}^{\prime}, h, \theta^{\prime}\right)>U\left(y_{i}^{\prime}, y_{-i}^{\prime}, h, \theta^{\prime}\right)\right]
$$

In this setting we now restate our main Theorem.

Theorem 2 Consider a common agency game $\Gamma_{M}$ in the class $\mathcal{G}$. If the preferences of the agent satisfy the $\theta$-Separability condition, then for every outcome that can be supported as a PBE of $\Gamma_{M}$, there is an equilibrium of the direct mechanism game $\Gamma_{\Theta}$ that implements the same outcome.

Proof. Let us consider the PBE denoted $\left(\left(\sigma_{i}^{*}\right)_{i \in N}, \sigma_{0}^{*}\right)$ of the game $\Gamma_{M} \in$ and let denote the corresponding agent's behavior at equilibrium by $\left(h^{*}, m^{*}\right)$. We will show that the outcome implemented by this equilibrium can be reproduced as an equilibrium of a game where principals' strategies are restricted to be direct mechanisms. 
Suppose that principals are playing their equilibrium strategies. For each contractible effort $h \in H$, we can consider the message sent by the agent, which satisfy

$$
\tilde{m}\left(\sigma_{i}^{*}, \sigma_{-i}^{*}, h, \theta\right) \in \underset{m \in M}{\arg \max } U\left(\sigma_{i}^{*}\left(m_{i}, h\right), \sigma_{-i}^{*}\left(m_{-i}, h\right), h, \theta\right), \forall \theta \in \Theta .
$$

Since the preferences of the agent satisfy the $\theta$-separability condition, the message $\tilde{m}_{i}($.$) turns out not to$ depend on $\theta$ for every $i \in N$. It will be only contingent on $\sigma_{i}^{*}, \sigma_{-i}^{*}$ and $h$. Formally: $\tilde{m}_{i}\left(\sigma_{i}^{*}, \sigma_{-i}^{*}, h\right)$.

The candidate for equilibrium in the take-it or leave-it offer game is the strategy profile $\left(\tilde{\sigma}_{i}(h), \tilde{\sigma}_{-i}(h), \tilde{\sigma}_{0}\right)$, where

$$
\begin{aligned}
& \forall i \in N, \forall h \in H \quad \tilde{\sigma}_{i}(h)=\sigma_{i}^{*}\left(\tilde{m}_{i}\left(\sigma_{i}^{*}, \sigma_{-i}^{*}, h\right), h\right), \\
& \forall\left(\sigma_{i}\right)_{i \in N} \in \tilde{\Sigma} \quad \tilde{\sigma}_{0} \in \underset{h \in H}{\arg \max } U\left(\left(\sigma_{i}(h)\right)_{i \in N}, h, \theta\right) .
\end{aligned}
$$

By construction the strategy profile $\left(\tilde{\sigma}_{i}(h), \tilde{\sigma}_{-i}(h), \tilde{\sigma}_{0}\right)$ induces the same outcome as the original PBE $\left.\left(\left(\sigma_{i}^{*}\right)_{i \in N}\right), \sigma_{0}^{*}\right)$.

We therefore have to show that this constitutes an equilibrium in the game $\Gamma_{\Theta}$. We first observe that $\tilde{\sigma}_{0}$ is the agent's best reply to the strategies $\tilde{\sigma}_{i}$. Then, we show that, given $\tilde{\sigma}_{-i}$, no principal $i$ has a profitable deviation.

Suppose that principal $i$ is deviating to $\sigma_{i}^{\prime} \neq \tilde{\sigma}_{i}$. A take-it or leave-it offer is, in fact, a particular indirect mechanism. The deviation $\sigma_{i}^{\prime}$ was, therefore, already available in the original game $\Gamma_{M}$. We can therefore show that whenever faced with the same deviation in $\Gamma_{M}$ and $\Gamma_{\Theta}$ the optimal response of the agent in terms of action will be the same.

If

$$
\left(h^{\prime}, m_{-i}^{\prime}\right) \in \underset{h, m}{\arg \max } U\left(\sigma_{i}^{\prime}(h), \sigma_{-i}^{*}\left(m_{-i}, h\right), h, \theta\right),
$$

then

$$
h^{\prime} \in \underset{h \in H}{\arg \max } U\left(\sigma_{i}^{\prime}(h), \tilde{\sigma}_{-i}(h), h, \theta\right), \forall i \in N .
$$

The proof is again given by contradiction. If this is not true, then there must exist a level $\bar{h}$ such that:

$$
U\left(\sigma_{i}^{\prime}(\bar{h}), \tilde{\sigma}_{-i}(\bar{h}), \bar{h}, \theta\right)>U\left(\sigma_{i}^{\prime}\left(h^{\prime}\right), \tilde{\sigma}_{-i}\left(h^{\prime}\right), h^{\prime}, \theta\right)
$$

We know that

$$
U\left(\sigma_{i}^{\prime}\left(h^{\prime}\right), \tilde{\sigma}_{-i}\left(h^{\prime}\right), h^{\prime}, \theta\right)=U\left(\sigma_{i}^{\prime}\left(h^{\prime}\right), \sigma_{-i}^{*}\left(\tilde{m}_{-i}\left(\sigma_{i}^{*}, \sigma_{-i}^{*}, h^{\prime}\right), h^{\prime}\right), h^{\prime}, \theta\right),
$$

and

$$
U\left(\sigma_{i}^{\prime}(\bar{h}), \tilde{\sigma}_{-i}(\bar{h}), \bar{h}, \theta\right)=U\left(\sigma_{i}^{\prime}(\bar{h}), \sigma_{-i}^{*}\left(\tilde{m}_{-i}\left(\sigma_{i}^{*}, \sigma_{-i}^{*}, \bar{h}\right), \bar{h}\right), \bar{h}, \theta\right) .
$$


Given the definition of $\tilde{m}_{i}($.$) and applying the \theta$-separability condition we get that:

$$
\tilde{m}_{-i}\left(\sigma_{i}^{*}, \sigma_{-i}^{*}, h^{\prime}\right) \in \underset{m_{-i} \in M_{-i}}{\arg \max } U\left(\sigma_{i}^{\prime}\left(h^{\prime}\right), \sigma_{-i}^{*}\left(m_{-i}, h^{\prime}\right), h^{\prime}, \theta\right) .
$$

As a result we have that $\tilde{m}_{-i}\left(\sigma_{i}^{*}, \sigma_{-i}^{*}, h^{\prime}\right)$ and $m_{-i}^{\prime}$ have to be payoff equivalent for the agent (both are optimal responses), and for the deviating principal. Using an argument similar to that developed in Claim 2 , this result follows directly from $\theta$-separability together with the restriction to games in the class $\mathcal{G}$.

Therefore:

$$
U\left(\sigma_{i}^{\prime}\left(h^{\prime}\right), \sigma_{-i}^{*}\left(\tilde{m}_{-i}\left(\sigma_{i}^{*}, \sigma_{-i}^{*}, h^{\prime}\right), h^{\prime}\right), h^{\prime}, \theta\right)=U\left(\sigma_{i}^{\prime}\left(h^{\prime}\right), \sigma_{-i}^{*}\left(m_{-i}^{\prime}, h^{\prime}\right), h^{\prime}, \theta\right)
$$

Hence, we get:

$$
U\left(\sigma_{i}^{\prime}(\bar{h}), \sigma_{-i}^{*}\left(\tilde{m}_{-i}\left(\sigma_{i}^{*}, \sigma_{-i}^{*}, \bar{h}\right)\right), \bar{h}, \theta\right)>U\left(\sigma_{i}^{\prime}\left(h^{\prime}\right), \sigma_{-i}^{*}\left(m_{-i}^{\prime}, h^{\prime}\right), h^{\prime}, \theta\right)
$$

But this contradicts the fact that:

$$
\left(h^{\prime}, m_{-i}^{\prime}\right) \in \underset{h \in H, m \in M}{\arg \max } U\left(\sigma_{i}^{\prime}(h), \sigma_{-i}^{*}\left(m_{-i}, h\right), h, \theta\right)
$$

The intuition behind this result is the following. Under $\theta$-separability, every deviation taken by principal $i$ in the indirect mechanism game can affect the message sent to principal $j$ only through a change in the action choice of the agent. Since the optimal response in terms of effort is the same in the two relevant games and it is contractible (observable), then there is no real informational loss in restricting to direct mechanisms. Note that this argument cannot be reproduced in a moral hazard setting. The reason is that the agent's effort choice is non-contractible and, hence, cannot be observed by the principals. As a result, the principal cannot extract any relevant information from the agent's action. Thus, if we restrict our attention to the externalities induced by multiple contracting, ${ }^{9}$ then, whenever agent's preferences are separable, direct mechanisms are enough to characterize all equilibria of the game.

As stated in the introduction, many common agency models considered in the literature satisfy this separability condition. Examples are given by Bernheim and Whinston (1986b); Grossman and Helpman (1994); Dixit, Grossman, and Helpman (1997); Laussel and Le Breton (1998). ${ }^{10}$ Our $\theta$-separability condition is also satisfied in some games of incomplete information (e.g. Le Breton and Salanie (2003)).

A key assumption of all these models is the absence of direct externalities among principals. That is, if we think of multiple sellers competing on the prices they are offering to a single agent, then the price

\footnotetext{
${ }^{9}$ That is, we disregard asymmetric information issues.

${ }^{10}$ Many models have been built on these seminal works and most of them satisfy our separability condition. The reader can refer to Aidt (1998); Rama and Tabellini (1998) and Laussel and Le Breton (2001), among many others. The same separability condition is also introduced in recent theoretical works on multi-principal multi-agent games (see Prat and Rustichini (2003)).
} 
charged by seller $i$ can be affected by the quantity the agent is buying from him, but not by the quantity bought from seller $j$. On the contrary, Bernheim and Whinston (1998) introduced a framework with direct externalities among principals in order to study exclusive dealing issues. Similarly, de Villemeur and Versaevel (2003) considered a model of Research and Development where every principal (a self interested firm) is directly affected by the decisions taken by the other principals.

Even though most of the popular works in common agency under complete information satisfy our separability requirement, they typically do not belong to the class $\mathcal{G} .{ }^{11}$ Thus, when analyzing this literature, one cannot in general disregard the existence of equilibrium outcomes of indirect mechanism games that cannot be supported in the corresponding direct mechanism games. If those equilibria exist, however, they will typically rely on a very particular form of indifference for the agent.

The following example illustrates in greater detail the features of this sort of equilibria. Consider a complete information game with two principals, where the agent takes no action. At equilibrium principals offer the menus $\bar{Y}_{1} \subset Y_{1}$ and $\bar{Y}_{2} \subset Y_{2}$. The agent chooses the items $y_{1}^{*}$ from $\bar{Y}_{1}$ and $y_{2}^{*}$ from $\bar{Y}_{2}$. If this outcome cannot be sustained by direct mechanisms, it means that when principals are offering $\left\{y_{1}^{*}\right\}$ and $\left\{y_{2}^{*}\right\}$ respectively, at least one of them should have a profitable deviation. Assume that principal 1 prefers playing $\left\{\tilde{y}_{1}\right\}$ than $\left\{y_{1}^{*}\right\}$ when principal 2 plays $\left\{y_{2}^{*}\right\}$. In the menu game, this deviation is not profitable, because there exist a $\left\{\bar{y}_{2}\right\}$ such that the agent weakly prefers the couple $\left(\tilde{y}_{1}, \bar{y}_{2}\right)$ to the couple $\left(\tilde{y}_{1}, y_{2}^{*}\right)$ and the principal 1 strictly prefers the couple $\left(y_{1}^{*}, y_{2}^{*}\right)$ to the couple $\left(\tilde{y}_{1}, \bar{y}_{2}\right)$. The agent must also (weakly) prefer $\left(y_{1}^{*}, y_{2}^{*}\right)$ to $\left(y_{1}^{*}, \bar{y}_{2}\right)$. For this to be compatible with our condition it has to hold that the agent is indifferent at least once, either between $\left(\tilde{y}_{1}, \bar{y}_{2}\right)$ and $\left(\tilde{y}_{1}, y_{2}^{*}\right)$ or between $\left(y_{1}^{*}, y_{2}^{*}\right)$ and $\left(y_{1}^{*}, \bar{y}_{2}\right)$, otherwise his preferences would not be separable. This means that simply by restricting principal 2 not to play $\bar{y}_{2}$ would be enough to eliminate the equilibrium that supports $\left(y_{1}^{*}, y_{2}^{*}\right)$. This example emphasizes the paradoxical features of equilibria that can only be supported by indirect mechanisms when the separability requirements are satisfied: If they exist, they turn out to be very sensible to slight perturbations in the principals' strategy spaces.

We have only considered environments with $\theta$-separable preferences because allowing for the action $h$ to be contractible introduces a new source of externality among principals. When the agent's preferences are type-dependent, a principal by changing his mechanism can induce a change, not only in the agent's effort choice, but also in the report of her private information to other principals. This interaction between the contractible action choice and the agent's private type introduces an essential problem of non-separability.

We now present an example showing that, in situations where agent's preferences are type-dependent, even the Strong Separability condition does not retrieve the Revelation Principle.

\footnotetext{
${ }^{11}$ The applied literature has mainly examined continuous games.
} 


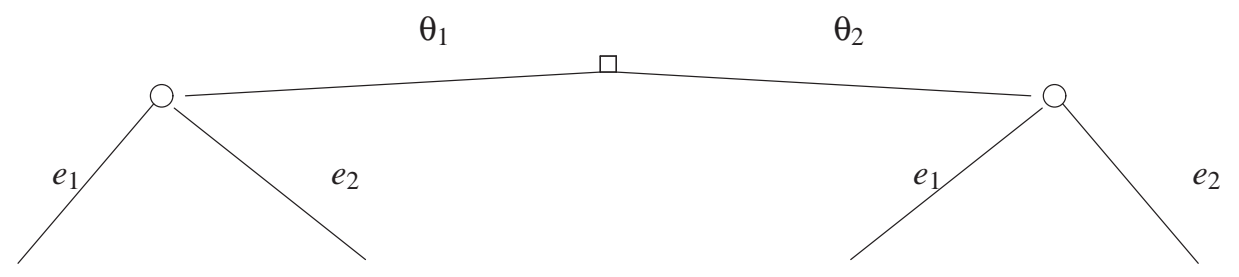

A

\begin{tabular}{|l|l|l|}
\hline A & $(3,3,1)$ & $(2,0,2)$ \\
\hline B & $\left(0,2, \frac{3}{2}\right)$ & $\left(1,-1, \frac{5}{2}\right)$ \\
\hline C & $\left(0,0, \frac{1}{2}\right)$ & $(0,0,1)$ \\
\hline
\end{tabular}

A

\begin{tabular}{|c|c|}
\hline$(0,0,2)$ & $(0,-1,3)$ \\
\hline$\left(0,0, \frac{21}{10}\right)$ & $\left(\frac{3}{2}, \frac{3}{2}, 4\right)$ \\
\hline$\left(0,0, \frac{1}{2}\right)$ & $(0,0,1)$ \\
\hline
\end{tabular}

B

\begin{tabular}{|c|c|c|}
\hline A & $(1,1,5)$ & $\left(0,0, \frac{3}{4}\right)$ \\
\hline B & $(0,0,6)$ & $(1,-1,1)$ \\
\hline $\mathrm{C}$ & $(0,0,7)$ & $\left(0,0, \frac{3}{2}\right)$ \\
\hline
\end{tabular}

B

\begin{tabular}{|c|c|c|}
\hline & A & B \\
\hline A & $\left(0,0, \frac{1}{2}\right)$ & $(-1,-1,0)$ \\
\hline B & $(-1,-1,0)$ & $\left(-2,2, \frac{5}{2}\right)$ \\
\hline $\mathrm{C}$ & $(-1,-1,4)$ & $\left(-1,-1, \frac{7}{2}\right)$ \\
\hline
\end{tabular}

In the example above the agent's type space is $\Theta=\left\{\theta_{1}, \theta_{2}\right\}$ and she is allowed to take the action $h \in H=\left\{h_{1}, h_{2}\right\}$, which is assumed to be contractible. There are two principals: principal 1 has the three available actions $\{A, B, C\}$, while principal 2 can choose between $A$ and $B$ only. The outcome $\left\{\left(\frac{3}{2}, \frac{3}{2}, 4\right)\right.$ if $\theta=\theta_{1},(1,1,5)$ if $\left.\theta=\theta_{2}\right\}$ cannot be sustained as an equilibrium in the direct mechanism game. If it were an equilibrium, then principals' strategies should involve the following behaviors: whenever the message $\theta_{1}$ is sent and $h_{2}$ is performed, then both principals play $B$; whenever the message $\theta_{2}$ is sent and $h_{1}$ is performed, they both play $A$.

Principal 2 has a profitable deviation in playing $B$ irrespectively of both the received message and the performed action. If he takes such a strategy, then the agent has an incentive to misreport her type and choose $h_{2}$ when she is $\theta_{2}$. In such a case principal 1 is playing $B$ and principal 2 earns a payoff of 2 instead of 1 .

We stress here that the same outcome can indeed be supported by allowing principals to offer menus. Let us consider the following strategy profiles: both principals play $A$ if $h_{1}$ is chosen; when the agent plays $h_{2}$, then principal 2 plays $B$ and principal 1 offers the menu $\{B, C\}$. If principal 2 deviates to a strategy that prescribes to play $B$ irrespectively of $h$, then the $\theta_{2}$ agent will play $h_{2}$ and ask for $C$ from principal 1, that makes the deviation unprofitable.

\section{Discussion}

We have identified a class of common agency games in which the Revelation Principle retains its power. Every allocation supported as a pure strategy equilibrium inside an arbitrarily large class of indirect mechanisms can therefore be implemented through simple direct mechanisms. 
To the best of our knowledge, an approach close to ours has only been followed by Peters (2003), who developed a similar intuition. His work is however focused on common agency games where the agent's private information does not affect her preference order. ${ }^{12}$ That is, incomplete information is not an issue in his analysis.

More precisely, Peters (2003) identifies a set of restrictions on players' preferences, called "noexternalities assumption", guaranteeing that every pure strategy equilibrium allocation of a common agency game with menus (i.e. indirect mechanisms) can in fact be supported at (a pure strategy) equilibrium in a simpler game where principals' strategies are simple take-it or leave-it offers. These restrictions can be resumed as follows: each principal's utility only depends on his own action and on the agent's choices; in addition, the agent has a weak preference ordering over the actions of every single principal that is independent of her decisions and of the other principals' offers.

In this paper we have shown how these conditions can be weakened considering finite generic games. Our analysis highlights that, when eliminating the possibility that the agent is indifferent among any array of contract offers, the scope of applicability for the Revelation Principle is widened.

Importantly, our results are robust to the introduction of direct externalities among principals. In addition, incomplete information can be embedded in the analysis, as there is no need to assume that the agent's preference ordering should be immune to changes in her private type. One should also observe that Peters' no-externalities assumption turns out not to be sufficient whenever moral hazard is explicitly considered: Attar, Piaser, and Porteiro (2006a) show that if the single agent is taken to be indifferent over alternative outcomes, there can be equilibrium allocations of an indirect mechanism game that cannot be sustained through simple direct mechanisms, even if the no-externalities assumption holds. We have hence proposed another strategy to establish the result, eliminating any source of indifference on the agent's side. $^{13}$

Finally, the case in which the agent's action is contractible is particulary interesting. Restricting to a scenario where agent's choices are fully observable allows us to isolate the external effects induced by competition over contracts. We have already emphasized that a relevant portion of the common agency literature has been built in a setting of complete information where the action taken by the agent is fully contractible for every principal. Interestingly, most of common agency models belonging to this class satisfy our separability condition. Our second condition, however, is likely not to be satisfied in those works: they typically involve a continuum of decisions and actions, while our condition is holds generically if the relevant sets are taken to be finite. ${ }^{14}$ Nevertheless, our results give arguments in favor of the restriction to direct mechanisms in any work where only our separability requirement is satisfied. If there exist equilibria that are sustainable through indirect mechanisms but not through direct ones, then they should necessarily rely on a very particular behavior of the agent when she is indifferent among two contract offers. If one assumes whenever indifferent the agent will take the "good" decision, then these

\footnotetext{
${ }^{12}$ See Peters (2003), p. 97.

${ }^{13}$ For a restatement of the no-externalities assumption in a moral hazard environment see Peters (2006). Attar, Piaser, and Porteiro (2006b) argue that our restrictions, as well as the modified Peters' ones, are hard to be satisfied in any existing research on common agency under moral hazard.

${ }^{14}$ See again Bernheim and Whinston (1986b); Grossman and Helpman (1994); Dixit, Grossman, and Helpman (1997); Laussel and Le Breton (1998); Aidt (1998); Rama and Tabellini (1998); Laussel and Le Breton (2001).
} 
equilibria will disappear. This indicates that they might be sensible to small changes in payoffs and they cannot therefore be approximated by any equilibrium of a finite generic game.

\section{Acknowledgment}

We are grateful to Eloisa Campioni, Claude d'Aspremont, Catarina Goulão, Pierre Fleckinger, Inés Macho-Stadler, Enrico Minelli, David Pérez-Castrillo, Uday Rajan and seminar participants at the ARC seminar at CORE, University of Padova, University of Venice, 2004 SED meeting in Palma and 2004 ASSET conference in Barcelona for helpful comments. All errors remain of course ours.

\section{References}

AIDT, T. (1998): "Political internalization of economic externalities and environmental policy," Journal of Public Economics, 69, 1-16.

Attar, A., E. Campioni, And G. Piaser (2005): "Multiple lending and constrained efficiency in the credit market," CORE DP 2005-31.

Attar, A., G. Piaser, And N. Porteiro (2006a): "Negotiation and take-it or leave-it in Common Agency with non-contractible actions," Journal of Economic Theory, forthcoming.

(2006b): "A note on Common Agency models of moral hazard," Universidad Pablo de Olavide, Mimeo.

Bernheim, B. D., And M. D. Whinston (1986a): “Common Agency,” Econometrica, 54(4), 923942.

(1986b): "Menu Auctions, Resource Allocation, and Economic Influence," Quaterly Journal of Economics, 101(1), 1-31.

(1998): “Exclusive Dealing,” Journal of Political Economy, 106(1), 64-103.

Biais, B., D. MARTIMORT, AND J.-C. ROCHET (2000): "Competing mechanisms in a common value environment," Econometrica, 78(4), 799-837.

Bisin, A., And D. Guaitoli (2004): "Moral hazard with non-exclusive contracts," Rand Journal of Economics, 2, 306-328.

De Villemeur, E., ANd B. Versaevel (2003): “Conflict and Cooperation on R\&D Markets," IDEI Working Paper.

Deaton, A., And J. Muellbauer (1980): Economics and Consumer Behaviour. Cambridge University Press.

Dixit, A., G. M. Grossman, And E. Helpman (1997): "Common agency and coordination: General theory and application to government policy making," Journal of Political Economy, 105(4), 752-769.

Epstein, L. G., And M. Peters (1999): “A revelation principle for competing mechanisms," Journal of Economic Theory, 88(1), 119-160. 
FISHBURN, P. C. (1970): Utility theory for decision making. New York: John Wiley \& Sons.

GibBARD, A. (1973): “Manipulation of voting schemes: A general result,” Econometrica, 41(4), 587601.

Grossman, G. M., And E. Helpman (1994): "Protection for sale," American Economic Review, 84(4), 833-850.

Ishiguro, S. (2005): “Competition and Moral Hazard,” Mimeo, Osaka University.

LAUSSEl, D., AND M. LE BRETON (1998): "Efficient private production of public goods under common agency," Games and Economic Behavior, 25, 194-218.

(2001): "Conflict and Cooperation: the structure of equilibrium payoffs in common agency," Journal of Economic Theory, 100, 93-128.

Le Breton, M., and F. SAlanie (2003): “Lobbying under political uncertainty," Journal of Public Economics, 87, 2589-2610.

Martimort, D. (1992): "Multi-principaux avec anti-selection,” Annales d'Economie et de Statistique, 0(28), 1-37.

(2004): "Delegated Common Agency under Moral Hazard and the Formation of Interest Group," mimeo, IDEI.

Martimort, D., And L. A. Stole (2001): "Common agency equilibria with discrete mechanisms and discrete types," CESifo Working Paper, n. 572.

(2002): “The revelation and delegation principles in common agency games," Econometrica, 70(4), 1659-1673.

(2003): “Contractual externalities and common agency equilibria," Advances in Theoretical Economics, 3(1).

Myerson, R. B. (1979): "Incentive compatibility and the bargaining problem,” Econometrica, 47, 6173.

Parlour, C. A., And U. RAJAn (2001): “Competition in Loan Contracts," American Economic Review, 91(5), 1311-1328.

PAVAN, A., AND G. CALZOLARI (2003): “A markovian revelation principle for common agency games,” mimeo Northwestern University.

PECK, J. (1997): "A note on competing mechanisms and the revelation principle," mimeo Ohio State University.

Peters, M. (2001): “Common Agency and the Revelation Principle,” Econometrica, 69(5), 1349-1372.

(2003): "Negotiation and take-it-or-leave-it in common agency," Journal of Economic Theory, 111(1), 88-109.

(2006): "Errata - Negotiation and take-it or leave-it in Common Agency," Journal of Economic Theory, forthcoming.

PIASER, G. (2005): “Stochastic and deterministic menus in common agency games," Economics Bulletin, 4(11), 1-6. 
PRAT, A., And A. Rustichini (2003): “Games played through agents,” Econometrica, 71, 989-1026.

RAma, M., And G. TABEllini (1998): "Lobbying by capital and labor over trade and labor market policies," European Economic review, 42, 1295-1316.

Rochet, J.-C. (1986): “Le contrôle des équations aux dérivées partielles issues de la théorie des incitations," Ph.D. thesis, Université Parix IX.

SEGAL, I., And M. D. Whinston (2003): "Robust predictions for bilateral contracting with externalities," Econometrica, 71(3), 757-791.

Strausz, R. (2003): "Deterministic Mechanisms and the Revelation Principle," Economic Letters, 79, 333-337. 